\title{
Socio-Economic Status and Exclusive Breastfeeding Among Infants in a Ugandan Cross-Sectional Study
}

\author{
Ratib Mawa ${ }^{1}$, Caroline Kambugu Nabasirye ${ }^{2}$, James Mulira $^{1}$, Catherine Nakidde ${ }^{1}$, \\ Frank Kalyango ${ }^{1}$, Dolorence Mary Angulo Alaki Wakida ${ }^{1}$, Margaret Chota ${ }^{1}$, \\ Tracy Leigh Schumacher ${ }^{3}$, Stephen Lawoko ${ }^{1}$, Krishna Nand Sharma ${ }^{1}$ \\ ${ }^{1}$ Department of Public Health, Faculty of Health Sciences, Victoria University, Kampala, Uganda \\ ${ }^{2}$ Department of Nursing and Midwifery, Faculty of Health Sciences, Lira University, Lira, Uganda \\ ${ }^{3}$ Department of Rural Health, Faculty of Health and Medicine, University of Newcastle, Newcastle, Australia
}

\section{Email address}

ratib@vu.ac.ug(R. Mawa), leakycarolyn326@gmail.com(C.K. Nabasirye),jmulira1@gmail.com(J. Mulira), kathy.lubowa@gmail.com(C. Nakidde),kalyangofrnk@gmail.com (F.Kalyango),dolwakida@gmail.co(D. M. A. A. Wakida), chotamargret@yahoo.com(M. Chota),tracy.schumacher@newcastle.edu.au(T.L.Schumacher), deanhealthscience@vu.ac.ug(S.Lawoko), vicechancellor@vu.ac.ug(K. N. Sharma)

\section{To cite this article:}

Ratib Mawa, Caroline Kambugu Nabasirye, James Mulira, Catherine Nakidde, Frank Kalyango, Dolorence Mary Angulo Alaki Wakida, Margaret Chota Tracy Leigh Schumacher, Stephen Lawoko, Krishna Nand Sharma. Socio-Economic Status and Exclusive Breastfeeding Among Infants in a Ugandan Cross-Sectional Study. Journal of Food and Nutrition Sciences. Vol. 7, No. 1, 2019, pp. 16-24. doi: $10.11648 /$ j.jfns.20190701.13

Received: March 28, 2019; Accepted: May 5, 2019; Published: June 3, 2019

\begin{abstract}
Nation-wide population based studies on socio-economic inequality in exclusive breastfeeding among infants is scarce in Uganda. This study examined the socio-economic inequality in exclusive breastfeeding among 1424 infants below 6 months of age in a nation-wide population based cross-sectional study. Self-reported maternal exclusive breastfeeding practice in the first six months of their last born baby's life was the outcome of interest. Household wealth index and maternal occupation constituted the proxy measures of socio-economic status, the exposure variable of interest. The distribution of exclusive breastfeeding practice by infant-mother dyad characteristics was calculated by conducting bivariate analysis. Multivariate binary logistic regression models were then fitted to calculate the odds ratios and their corresponding $95 \%$ confidence intervals for exclusive breastfeeding by maternal occupation and household wealth index. The results showed that half of the infants were boys, close to half of their mothers were farmers, and $23 \%$ and $16.9 \%$ of the infants lived in the poorest and richest households respectively. Overall $67 \%$ of the infants were exclusively breastfed. The odds ratios for exclusive breastfeeding by maternal occupation were $0.62(0.26-1.50)$ for infants whose mothers were professionals $/$ technical $/ \mathrm{managers,}$ 0.97(0.50-1.87) for clerical/sales, 0.78 (0.51-1.21), for Farmers, 0.72 (0.29-1.82) for Household/Domestic/Services, 0.72 (0.391.36) for skilled manual workers and $0.72(0.25-2.02)$ for unskilled manual workers compared to infants of non-working mothers. The odds ratios for exclusive breastfeeding by household wealth index were 2.38 (1.30-4.33), for the poorest, 2.16 (1.18-3.96) poorer, 1.91 (1.10-3.48) middle, and 1.41 (0.75-2.64), for richer households compared to infants in the richest households. In conclusion, an inverse relationship was found between household socio-economic status and exclusive breastfeeding among infants below six months of age and inadequate evidence to conclude existence of an association between maternal socio-economic status and exclusive breastfeeding. Targeting exclusive breastfeeding interventions to mother-infant dyad living in affluent families might be important in reducing socio-economic inequality in exclusive breastfeeding among infants below six months of age in Uganda.
\end{abstract}

Keywords: Exclusive Breastfeeding, Socio-Economic Status, Infants, Uganda 


\section{Introduction}

The importance of exclusive breastfeeding to infantmother dyad cannot be over-emphasized. It's one of the known nutrition specific interventions that contributes to reduced infant morbidity and mortality especially from infectious diseases and protects against childhood obesity and some chronic conditions in later life [1-4]. Following the Innocenti declaration on the protection, promotion and support for optimal breastfeeding practice across the globe [5], and its subsequent modification by the World Health Organization (WHO) [6], a lot of effort was made at both national and international level to promote optimal breastfeeding practices but yielded moderate results [7]. The global breastfeeding scorecard for 2018 indicated that less than half $(41 \%)$ of the infants below six months of age were exclusively breastfed globally [8]. For low and middle income countries some estimates showed very low prevalence of exclusive breastfeeding (37\%) among infants below six months of age compared to the total global prevalence of exclusive breastfeeding [2]. In sub-Saharan Africa, estimates based on data from nationally representative demographic and health surveys conducted between 2010-2015 in 29 sub-Saharan African countries showed that exclusive breastfeeding prevalence among infants below six months of age ranged from as low as $23.7 \%$ to $56.6 \%$ [9]. Although Uganda was ranked among the 23 countries included in the Global Breastfeeding Collective group scorecard for 2017 for having achieved the UNICEF/WHO 2025 exclusive breastfeeding prevalence target of $50 \%$ [10], 34\% of her infants below six months of age are still not exclusively breastfed [11]. This implies, that proportion of infants (exclusively breastfed) are not reaping the benefits of exclusive breastfeeding and might undermine the efforts to reduce infant and under-five mortality in Uganda [11], as well as the achievement of the United Nation 2030 sustainable development goals [12] and the new 2030 UNICEF/WHO global exclusive breastfeeding prevalence target of $70 \%$ [13].

Social and behavioral theories have been suggested to explain exclusive breastfeeding practice among women. This include the extended theory of planned behavior $[14,15]$ and Bourdieu's social theory, which proposed that 'meaning and experiences' influenced by social circumstances in which people live (lay knowledge) as opposed to attitudes' or 'beliefs might be important in explaining infant feeding practices among women living in various social environments [16, 17]. Indeed infant feeding practices vary between societies and communities within the same society, hence the multi-causal nature of the default in exclusive breastfeeding practice. Substantial evidence exists on the association between low socio-economic status and negative health outcomes [18] in both children and adults. Socioeconomic inequalities in exclusive breastfeeding of infants have been reported in both developing and developed countries, however the direction and the magnitude of the association between socio-economic status and exclusive breastfeeding of infants differs between developed and developing countries [19]. In sub-Saharan Africa, a systematic review of Kenyan studies showed that employed mothers were less likely to exclusively breastfeed their infants compared to non-working mothers, and household food insecurity was associated with less likelihood of exclusive breastfeeding practices among women [20]. On the same note, a population based cross-sectional study in Ethiopia, found that unemployed mothers were 9 times more likely to exclusively breastfeed compared to employed mothers [21]. On the contrary a Nigerian cross-sectional study found no association between maternal occupation (work status) and exclusive breastfeeding [22] however this study included all occupational groups into one category hence misclassification of the subjects with respect to exposure and residual confounding cannot be ruled out. In studies where socio-economic status was measured using formal educational attainment, Mothers who reported having attained primary level education were found to be less likely to exclusively breastfeed their infants in the first six months compared to those that reported having attained tertiary level education [22]. The challenge of using proxy measures of socio-economic status in assessing health inequalities in developing countries has been discussed elsewhere [23]. As a result the validity of education and occupation in measuring household and individual income has been questioned hence the development of the household wealth index commonly applied in demographic and health surveys in developing countries. Therefore the discrepancies in results of studies that assessed the effect of maternal socio-economic status on exclusive breastfeeding practices using the above proxy measures are expected.

Few epidemiological studies investigated the relationship between socio-economic status and exclusive breastfeeding of infants below six months of age in Uganda. A more recent population based cross-sectional study based on secondary data from 2006 and 2011 Uganda Demographic and Health Surveys (UDHS) found inadequate evidence of an association between maternal socio-economic status and exclusive breastfeeding practice in the year 2006, however infants in the $60 \%$ affluent households were less likely to be exclusively breastfed compared to their counterparts in less affluent families. The same study did not find evidence of an association between maternal formal education, occupation and exclusive breastfeeding among infants, when data for 2011 UDHS was analyzed [24]. These findings are inconsistent given the short duration of the two periods for data collection and the expected changes in socio-economic status relative to Uganda's economic growth in the 5 year period (2006-2011). In another Ugandan cross-sectional study based on the 2006 UDHS, an inverse relationship between household socio-economic status and exclusive breastfeeding among infants less than 6 months of age was found, although the findings of this study agrees with the earlier described Ugandan study, there were inconsistencies in the statistical analysis and the measure of effect reported were not clear, making the validity of the results questionable 
[25]. Another cross-sectional study conducted in Ugandan district of Rakai over a decade ago reported only the crude odds ratios for exclusive breastfeeding by maternal formal education and occupation but no evidence of association was found, this makes the conclusion of existence or nonexistence of association between socio-economic status and exclusive breastfeeding invalid because potential confounders were not adjusted for [26].

Other factors that have been reported to be positively associated with exclusive breastfeeding among infants below six months of age in sub-Saharan Africa and other developing countries include maternal postnatal care attendance, births attended to by healthcare workers, absence of maternal breast conditions/disease [27], living in rural area, maternal none smoking habits, multi-parity, infant gestational age of $>=37$, supportive husbands and motherinfant bed sharing when sleeping [28]. Last but not the least, psychosocial factors such as maternal attitude towards breastfeeding, subjective norms and practice control were also found to positively or negatively affect exclusive breastfeeding practices of mothers [29]. In general most factors identified to be influencing exclusive breastfeeding practice tend to rotate around culture, social environment, medical, and maternal social position and socio-economic status. Identifying socio-economic inequalities in exclusive breastfeeding of infants below six months of age is essential for developing evidence based optimal breastfeeding promotion interventions and achieving the WHO goal of health equity (Health for all). Therefore this study aimed to examine the association between socio-economic status and exclusive breastfeeding among infants below six months of age in the general population in Uganda.

\section{Methods and Materials}

\subsection{Setting and Materials}

The materials for this study were obtained from Uganda, a developing country located in East Africa with 2019 projected population of 40 million people [30]. Uganda still has a weak healthcare system financed by a combination of tax and donor funds. The healthcare system has three operational pathways consisting of private not for profit, private for profit and public health care services, however there also exists private for profit services within the public healthcare system especially at the national referral hospital. Nutrition interventions (both curative and preventive services) are integrated into the existing government and private-not-for-profit healthcare services provided at the lowest health unit (health center II) to the highest level health unit (national referral hospital). This study was based on secondary data for the 2016 Uganda Demographic and Health Survey (UDHS) sponsored by USAID. The 2016 UDHS is the $6^{\text {th }}$ in a series of five-year interval surveys that started since 1988/89. It utilized a two-stage cluster sampling method to select a household sample nation-wide from which data on health and social issues of national importance were collected. In the first stage, the 2014 Uganda census enumeration areas were selected and in the second stage a random sample of households were then selected. In each selected household, women aged 15-49 years and a subsample of men aged 18-54 years was interviewed. Standardized structured questionnaires were dispensed through face-to-face interviews by trained enumerators, anthropometry and biochemical test for specific health indicators were also conducted by trained and experienced medical professionals. The response rate for both women and men was very high $97 \%$ and $94 \%$ respectively. For more details on the sampling procedure applied, data collection tools and processes, survey response rate, please refer to the 2016 UDHS final report [11].

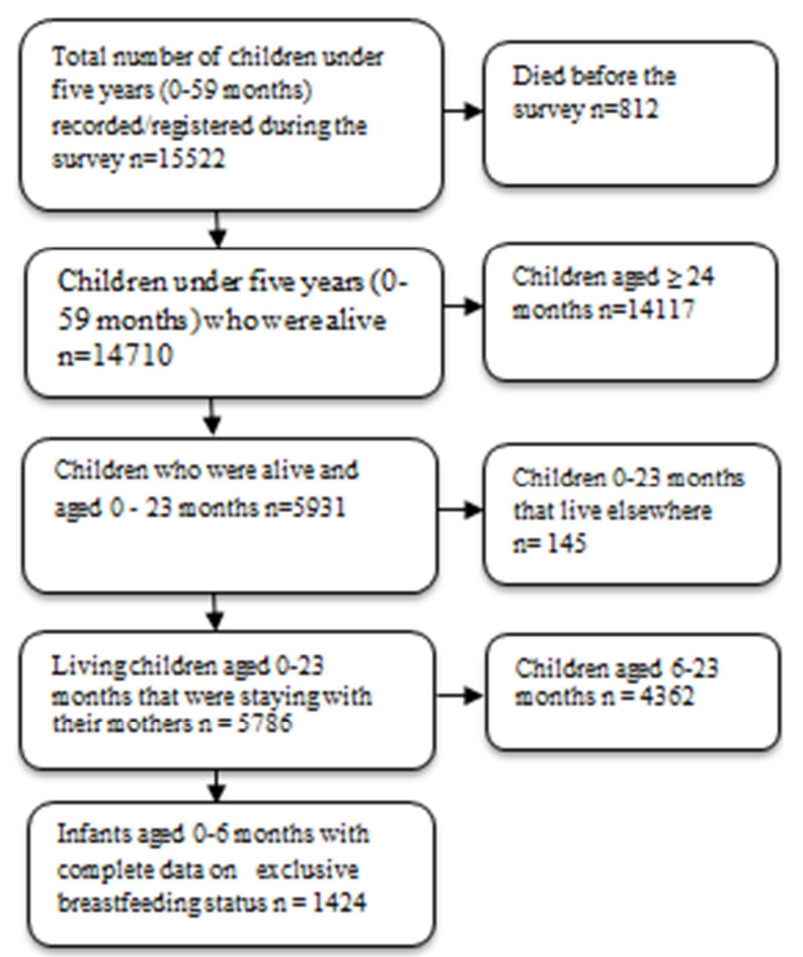

Figure 1. Schematic representation of the selection process of the study population.

\subsection{Study Population and Design}

A population based cross-sectional study based on the 2016 UDHS children's dataset obtained for Demographic and Health Survey program. A total of 1424 (weighted=1389) infants less than 6 months who were alive and whose mothers participated in survey were examined at the time of the 2016 UDHS. This mother-infant dyad was chosen based on the WHO recommendation/guidelines on infant and young child feeding practices [31]. Although HIV prevalence in the general population remained high $(7.3 \%)$ and mothers who are HIV positive can opt for alternative feeding options for their infants below six months [32], Uganda's policy on IYCF recommends all infants less than 6 months to be exclusively breastfeed unless replacement feeding is acceptable, feasible, affordable, sustainable and safe [33]. 


\subsection{Exposure and Outcome}

\subsubsection{Socio-Economic Status}

Household wealth index and maternal occupation as proxy measures of socio-economic status at household and individual level were the exposure variables of interests. These self-reported proxy measures of socio-economic status were measured using standardized structured questionnaires. Household wealth index, a measure of a household's cumulative standard of living, was calculated based on households ownership of selected assets. Data on household assets was collected using the household questionnaire which included questions on household ownership of properties such as cars and televisions, housing characteristics such as floor, wall, roofing materials etc, drinking water sources, toilet facilities, etc. Each household item for which data was collected was then assigned a factor score generated through principle component analysis. The scores were then summed up and individuals were ranked according to the score of their households. The sample was then divided into population quintiles ranking households into in the $20 \%$ poorest, poorer, middle, and richer to the top $20 \%$ richest households. Readers may refer to the 2016 UDHS report for details on the construction of the household wealth index variable [11]. By answering the question; what is your occupation? (That is, what kind of work do you mainly do?), mothers stated their occupations, recorded according to international classification of occupations [34]. For suitability of analysis, mothers were grouped into seven occupational categories that have similar earnings in Ugandan context; Not working, (not in the labor force), professional/technical/managerial, clerical and sales, agriculture (self-employed \& employee), household/domestic/services providers, skilled manual and unskilled manual workers. The occupational category not working (not in the labor force) was used as the reference category.

\subsubsection{Exclusive Breastfeeding}

Maternal self-reported exclusive breastfeeding of their infants was defined as having fed their infant on breast milk only in the 24 hours proceeding the interview day in the 2016 UDHS. This definition was derived from the World Health Organization's (WHO) Infant and Young Child Feeding Practices indicators [35]. It was measured using the standardized women's questionnaire. Mothers were asked a series of questions about their infant feeding practices in the last 24 hours preceding the survey day. Did you ever breastfeed (NAME)? In the first three days after delivery, was (NAME) given anything to drink other than breast milk? Are you still breastfeeding (NAME)? Did (Name) drink or eat? a) Plain water? b) Fresh fruit juice or juice concentrate? c) Clear broth? d) Milk such as tinned, powdered, or fresh animal milk? e) Infant formula? f) Any other liquids? g) Yogurt? h) Cheese or other foods made from milk? i) Any commercially fortified baby food such as cerelac? k) Pumpkins,
Pumpkin, carrots, squash, or sweet potatoes that are yellow or orange inside? 1) Cassava, yams (Juuni, Ndaggu, Baluggu), white sweet potatoes, Irish potatoes, manioc or any other roots or tubers?, m) Banana (Matooke, Ndiizi, Gonja)? n) Any dark green, leafy vegetables (dodo, nakati, spinach, amaranth, bugga, sunsa, jobyo, marakwang, sukuma wiki, Nsugga, Ggobe, Timpa)? o) Ripe mangoes, or pawpaws? p) Any other fruits or vegetables (passion fruit, jack fruit, pineapple, oranges, sugarcane)? q) Liver, kidney, heart, or other organ meats? r) Any beef, pork, lamb or goat, including products made from these meats (kebabs, sausages, chaps)? s) Any chicken, duck, turkey, pigeon, or other poultry? t) Eggs (from chickens, ducks or other poultry)? u) Fresh or dried fish or silver fish (mukene, kenje)? v) Any foods made from beans, peas, lentils, or nuts? w) Any sugary foods such as chocolates, sweets, candies, pastries, cakes or biscuits? x) Any cooking oil, margarine, butter or other oils/fats? y) Any other solid, semi-solid, or soft food? Maternal responses to the above questions were recorded in the 2016 UDHS dataset as yes or no. Then a binary variable exclusive breastfeeding was created and recorded as 1 if the infant was exclusively breastfed in the last 24 hours preceding the survey and 0 otherwise.

\subsection{Confounding}

The following variables were included as potential confounding variables based on published literature on their association with exclusive breastfeeding among infants below six months of age. Infants sex, age (months), birth weight (grams: two categories $\geq 2500$ and less than $2500 \mathrm{~g}$ ), mode of delivery (two categories: vaginal delivery, caesarean section), place of delivery (three categories: home, health facility, others), maternal age (years), maternal education level (four categories no education, primary, secondary and tertiary), maternal marital status (four categories; never married, married, living with a partner, widow/divorced/separated) paternal educational level (four categories; no education, primary, secondary and tertiary level), and place of residence (urban Vs rural). Information on these potential confounding variables was collected using the standardized 2016 UDHS questionnaires. Refer to the 2016 UDHS final report for details of the questionnaires used for data collection.

\subsection{Statistical Analysis}

To calculate the distribution of the study subjects by socio-demographic characteristics and exclusive breastfeeding status, bivariate analysis was conducted. To calculate the crude and adjusted odds ratios and their corresponding 95\% confidence intervals for exclusive breastfeeding by household wealth index and maternal occupation, univariate and multivariate binary logistic regression models were fitted. Only potential confounding variables with significant contribution to the models were included. To account for clustering and stratification introduced by the study design, complex survey data 
analysis techniques were applied. All infants with missing values for either outcome or exposure variables were excluded from the analysis. The statistical analysis was conducted using SPSS version 24.

\subsection{Ethical Consideration}

The survey procedures and questionnaires were reviewed and ethical approval was done by ICF International (Calverton, MD, USA) Institutional Review Board (IRB). Before the data collection exercise, women and men that participated in the survey provided a written informed consent. To ensure privacy, data obtained from participants was recorded in an anonymous manner hence individual information was un-identifiable. The use of the secondary data for the purpose is research was approved by DHS program archivist, authorization number 117079.

\section{Results}

As shown on Table 1, half of the infants were girls, 5.8\% had low birth weight, slightly more $(35.9 \%)$ were in the age group of 2-3 years, majority (76.2\%) of them were delivered in health facilities, and the majority $(35.9 \%)$ were aged $2-3$ months. Most of the infants had mothers (62.60\%) who reported having attained primary level education and the majority $(47.30 \%)$ of had mothers that reported living with a male partner. Occupational distribution of the infants mothers showed that close to half $(46.7 \%)$ of their mothers were employed in the agricultural sector (Farmers) and the lowest proportion $(2.6 \%)$ of their mothers identified their occupation as unskilled manual. The distribution of infants by paternal formal education was similar to that of their mothers. Majority $(54.4 \%)$ of the infants had fathers who attained primary level education and the least proportion (5.9\%) had fathers with no formal education. Similar percent distribution of infants by household socio-economic status was found with slightly more $(23 \%)$ of the infants were living in the poorest households and the lowest proportion (16.9\%) in lived in the richest households. Most $(82.6 \%)$ of the infants had mothers that resided in rural areas.

The prevalence of exclusive breastfeeding of infants aged 0.5 months was $67 \%$. However in general the distribution of the prevalence of exclusive breastfeeding was similar across all socio-demographic characteristics of the infants. Notably infants aged $0-1$ month had the highest $(81.5 \%)$ and those aged 4-5 months had the lowest $(50 \%)$ prevalence of exclusive breastfeeding. Girls compared to boys also had slightly high prevalence of exclusive breastfeeding $(68.8 \%$ Vs $65.2 \%$ ). Low birth weight infants also had higher prevalence $(69.1 \%)$ than infants that had normal birth weights $(66.8 \%)$. Infants whose mothers were reported not having been married had the highest prevalence $(72.8 \%)$ and those whose mothers were living with a male partner had the lowest prevalence $(65 \%)$ of exclusive breastfeeding. By place of delivery, infants born in other places other than a health facility and at home had the highest prevalence (69\%) and those born in health facilities had the lowest prevalence $(66.7 \%)$ of exclusive breastfeeding. The distribution of the prevalence of exclusive breastfeeding was also similar across maternal age groups. However older mothers in the age group of 40-49 years had the highest (75.6\%) prevalence of exclusive breastfeeding and those in the age group of 25-29 years had the lowest $(62.7 \%)$ prevalence of exclusive breastfeeding. Similar distribution of the prevalence of exclusive was observed across maternal education. However infants whose mothers had primary level education had the highest $(67.9 \%)$ prevalence of exclusive breastfeeding and those whose mothers had tertiary level education had the lowest prevalence (64.3\%) of exclusive breastfeeding. Maternal occupational distribution of the prevalence of exclusive breastfeeding showed that infants whose mothers were skilled manual workers had the lowest prevalence (57.7\%) and those that did clerical/sales job had the highest prevalence $(73.2 \%)$ of exclusive breastfeeding. The distribution of the prevalence of exclusive breastfeeding by paternal education also showed that infants whose fathers had no formal education had the highest $(69.60 \%)$ and those whose fathers had tertiary level education had the lowest (63.6\%) prevalence of exclusive breastfeeding. Infants in the poorest and poorer households had the highest prevalence of exclusive breastfeeding i. 71.2 and $70.90 \%$ respectively while those in the richer households had the lowest prevalence of exclusive breastfeeding. Rural-urban distribution in the prevalence of exclusive breastfeeding was similar (67.0 Vs 67.2\%). Lastly infants whose mothers had no health insurance had slightly higher prevalence $(67.1 \%)$ than their counterparts whose mothers had health insurance $(60.9 \%)$.

The crude and adjusted odds ratios for excusive breastfeeding by maternal occupation and household wealth index are shown on Table 2 and Table 3 respectively. Compared to infants whose mothers were not working, reduced odds ratios for exclusive breastfeeding was found with infants whose mothers had who professional/technical/managerial, clerical/sales, agricultural, household/domestic/services skilled manual and unskilled manual work having OR; 0.68, 1.22, 0.95, $0.65,0.60,1.2$ respectively. However after adjusting for potential explanatory variables, the odds ratios either increased or decreased to $0.62,0.97,0.78,0.72,0.72$, and 0.72 respectively. While the crude odds ratios for exclusive breastfeeding for infants in the poorest, poorer, middle and richer households compared to the richest households were $1.46,1.43,1.20$ and 0.88 respectively. When adjusted for important confounding variables, the odds ratios increased to $2.38,2.16,1.91$, and 1.41 for infants in the poorest, poorer, middle and richer households respectively. 
Table 1. Socio-demographic characteristics of 1424 (weighted 1389) infants aged 0-6 months by exclusive breastfeeding status.

\begin{tabular}{|c|c|c|c|}
\hline \multirow{2}{*}{ Characteristics } & \multirow{2}{*}{ Total (\%) } & \multicolumn{2}{|c|}{ Exclusive breastfeeding status } \\
\hline & & Not exclusively breastfed & Exclusively breastfed \\
\hline \multicolumn{4}{|l|}{ Child sex } \\
\hline Male & $690(49.70)$ & $240(34.80)$ & $450(65.20)$ \\
\hline Female & $699(50.30)$ & $218(31.20)$ & $481(68.80)$ \\
\hline Total & $1389(100)$ & $458(33.00)$ & $931(67.00)$ \\
\hline \multicolumn{4}{|l|}{ Birth weight (grams) } \\
\hline$<2500$ & $81(5.80)$ & $25(30.90)$ & $56(69.10)$ \\
\hline$\geq 2500$ & $1308(94.20)$ & $434(33.20)$ & $874(66.80)$ \\
\hline \multicolumn{4}{|l|}{ Child`s age (years) } \\
\hline $0-1$ & $439(31.60)$ & $81(18.50)$ & $358(81.50)$ \\
\hline $2-3$ & $499(35.90)$ & $152(30.50)$ & $347(69.50)$ \\
\hline $4-5$ & $452(32.50)$ & $226(50.00)$ & $226(50.00)$ \\
\hline \multicolumn{4}{|l|}{ Place of delivery } \\
\hline Home & $301(21.70)$ & $97(32.20)$ & $204(67.80)$ \\
\hline Health facility & $1059(76.20)$ & $353(33.30)$ & $706(66.70)$ \\
\hline Others & $29(2.10)$ & $9(31.00)$ & $20(69.00)$ \\
\hline \multicolumn{4}{|l|}{ Maternal age (years) } \\
\hline $15-19$ & $199(14.30)$ & $72(36.20)$ & $127(63.80)$ \\
\hline $20-24$ & $445(32.00)$ & $124(27.90)$ & $321(72.10)$ \\
\hline $25-29$ & $319(22.90)$ & $119(37.30)$ & $200(62.70)$ \\
\hline $30-34$ & $243(17.50)$ & $88(36.20)$ & $155(63.80)$ \\
\hline $35-39$ & $143(10.30)$ & $46(32.20)$ & $97(67.80)$ \\
\hline $40-49$ & $41(2.90)$ & $10(24.40)$ & $31(75.60)$ \\
\hline \multicolumn{4}{|l|}{ Maternal Education } \\
\hline No education & $136(9.80)$ & $45(33.10)$ & $91(66.90)$ \\
\hline Primary & $870(62.60)$ & $279(32.10)$ & $591(67.90)$ \\
\hline Secondary & $286(20.60)$ & $100(35.00)$ & $186(65.00)$ \\
\hline Tertiary & $98(7.10)$ & $35(35.70)$ & $63(64.30)$ \\
\hline \multicolumn{4}{|l|}{ Maternal Marital Status } \\
\hline Never married & $81(5.80)$ & $22(27.20)$ & $59(72.80)$ \\
\hline Married & $542(39.00)$ & $174(32.10)$ & $368(67.90)$ \\
\hline Living with a partner & $657(47.30)$ & $230(35.00)$ & $427(65.00)$ \\
\hline Widow/Divorced/Separated & $109(7.80)$ & $32(29.40)$ & $77(70.60)$ \\
\hline \multicolumn{4}{|l|}{ Maternal occupation } \\
\hline Not in labor force & $282(20.30)$ & $87(30.90)$ & $195(69.10)$ \\
\hline Professional/Technical/Managerial & $78(5.60)$ & $31(39.70)$ & $47(60.30)$ \\
\hline Clerical/Sales & $127(9.10)$ & $34(26.80)$ & $93(73.20)$ \\
\hline Agriculture (self-employed/employee) & $649(46.70)$ & $207(31.90)$ & $442(68.10)$ \\
\hline Household/domestic/services & $69(5.00)$ & $28(40.60)$ & $41(59.40)$ \\
\hline Skilled manual & $149(10.70)$ & $63(42.30)$ & $86(57.70)$ \\
\hline Unskilled manual & $36(2.60)$ & $10(27.80)$ & $26(72.20)$ \\
\hline \multicolumn{4}{|l|}{ Paternal Education level } \\
\hline No education & $69(5.90)$ & $21(30.40)$ & $48(69.60)$ \\
\hline Primary & $633(54.40)$ & $214(33.80)$ & $419(66.20)$ \\
\hline Secondary & $321(27.60)$ & $102(31.80)$ & $219(68.20)$ \\
\hline Tertiary & $140(12.00)$ & $51(36.40)$ & $89(63.60)$ \\
\hline \multicolumn{4}{|l|}{ Household Wealth index } \\
\hline Poorest & $320(23.00)$ & $92(28.80)$ & $228(71.20)$ \\
\hline Poorer & $306(22.00)$ & $89(29.10)$ & $217(70.90)$ \\
\hline Middle & $275(19.80)$ & $90(32.70)$ & $185(67.30)$ \\
\hline Richer & $254(18.30)$ & $101(39.80)$ & $153(60.20)$ \\
\hline Richest & $235(16.90)$ & $87(37.00)$ & $148(63.00)$ \\
\hline \multicolumn{4}{|l|}{ Type of place of residence } \\
\hline Urban & $241(17.40)$ & $79(32.80)$ & $162(67.20)$ \\
\hline Rural & $1147(82.60)$ & $379(33.00)$ & $768(67.00)$ \\
\hline
\end{tabular}

Note: weighted totals might not add-up to the sample size across different characteristics due to missing values. NA= Not available 
Table 2. Crude and adjusted odds ratio (OR) and 95\% Confidence Interval (CI) for exclusive breastfeeding among 1389 (Un-weighted 1424) infants aged 0-6 months by maternal occupation

\begin{tabular}{lll}
\hline \multirow{2}{*}{ Maternal Occupation } & \multicolumn{2}{l}{ Exclusive breastfeeding for infants aged 0-5 months } \\
\cline { 2 - 3 } & Crude OR (95\% CI) & Adjusted OR (95\% CI) \\
\hline Not working & 1.00 & 1.00 \\
Professional/Technical/Managerial & $0.68(0.45-1.32)$ & $0.62(0.26-1.50)$ \\
Clerical / Sales & $1.22(0.71-2.08)$ & $0.97(0.50-1.87)$ \\
Agricultural work & $0.95(0.67-1.35)$ & $0.78(0.51-1.21)$ \\
Household / Domestic / Services & $0.65(0.34-1.25)$ & $0.72(0.29-1.82)$ \\
Skilled Manual & $0.60(0.38-0.96)$ & $0.72(0.39-1.36)$ \\
Unskilled Manual & $1.22(0.52-2.83)$ & $0.72(0.25-2.02)$ \\
\hline
\end{tabular}

OR Adjusted for maternal age, maternal education level, Place of Delivery, Child`s gender, Child`s Age, Paternal Education, Health Insurance, time for initiation of breastfeeding after birth, Type of Place Residence, household wealth index.

Table 3. Crude and adjusted odds ratio (OR) and their corresponding 95\% Confidence Intervals (CI) for exclusive breastfeeding among 1389 (Un-weighted 1424) infants aged 0-6 months by household socio-economic status.

\begin{tabular}{lll}
\hline \multirow{2}{*}{ Household Wealth Index } & \multicolumn{2}{l}{ Exclusive breastfeeding for infants aged 0-5 months } \\
\cline { 2 - 3 } & Crude OR (95\% CI) & Adjusted OR (95\% CI) \\
\hline Richest & 1.00 & 1.00 \\
Poorest & $1.46(0.95-2.24)$ & $2.38(1.30-4.33)$ \\
Poorer & $1.43(0.95-2.16)$ & $2.16(1.18-3.96)$ \\
Middle & $1.20(0.77-1.88)$ & $1.91(1.10-3.48)$ \\
Richer & $0.88(0.56-1.40)$ & $1.41(0.75-2.64)$ \\
\hline
\end{tabular}

OR Adjusted for child sex, child age, birth weight, maternal age, maternal education level, occupation mother, paternal education, type of place residence

\section{Discussion}

The findings of this study showed high prevalence (67\%) of exclusive breastfeeding among infants below six months of age. This is above the global average of $41 \%$ reported by UNICEF/WHO in their most recent breastfeeding scorecard of 2018 [8] and slightly above the Kenyan exclusive breastfeeding rate of $61 \%$ in their 2014 demographic and health survey [36] as well as that of Tanzania's 59\% in $2015 / 2016$ [37], though these are not standardized prevalence of exclusive breastfeeding to necessitate direct comparison. Nevertheless the country has achieved the WHO/UNICEF 2025 exclusive breastfeeding prevalence target of $50 \%$ among infants below six months of age [10], but fell short of the new UNICEF/WHO exclusive breastfeeding target of $70 \%$ by the year 2030 [8].

When socio-economic status was assessed using maternal occupation, the results showed that infants whose mothers had professional/technical/managerial occupation were $38 \%$, clerical $/$ sales $3 \%$, agriculture $22 \%$, household $/$ domestic/services $28 \%$, skilled manual $28 \%$, and unskilled manual $28 \%$ less likely to be exclusively breastfed compared to those whose mothers were not working (not in the labor force) at the time of conduct of the 2016 UDHS. However there was inadequate evidence on the existence of an association between maternal occupation and exclusive breastfeeding of infants below six months of age. This might be due to the weakness of occupation as a proxy measure of socio-economic status in low and middle income countries [23], where formal occupations are scarce and casual labor plus small household enterprises are the most common sources of income that are often unstable [38]. Secondly the comparator i. e. mothers not in the labor market might be a mixture of mothers from different occupational groups with differential causal pathways to exclusive breastfeeding practices leading to under or over estimate of the effect measure. However the findings are in agreement with those of a Ugandan cross-sectional study that found no association between maternal occupation and exclusive breastfeeding among infants below six months of age [24] and that of an Ethiopian study that found a positive association between maternal unemployment and exclusive breastfeeding [21]. However this finding differed from those of a Kenyan systematic review that found a positive association between maternal occupation and exclusive breastfeeding of infants below six months of age [20]. These disparities in the relationship between maternal occupation and exclusive breastfeeding might need further investigation to ascertain whether maternal occupation has an independent effect on exclusive breastfeeding practice as opposed to its being a proxy measure of socio-economic status or some effect medication might by the different environments in which Ugandan infantmother dyads.

When household wealth index (proxy measure of household socio-economic status) was used in the analysis as the exposure variable, adjusted for important confounders, we found an association between household socio-economic status and exclusive breastfeeding of infants below six months of age. The results showed that infants in the poorest and poorer households were 1.38 and 1.16 times more likely to be exclusively breastfeed compared to those that lived in the richest households. Likewise infants that lived in the middle and richer households were 0.91 and 0.41 times more likely to be exclusively breastfed compared to infants in the richest households. These findings might be because the less affluent families have less financial and physical access to baby formulas, and less access to information on alternative feeding options for infants given that majority of the motherinfant dyads stay in rural areas of Uganda. On the other, the high prevalence of antenatal care attendance among Ugandan 
women might have contributed to increased maternal knowledge on optimal breastfeeding practices, of which knowledge might have been translated into practice after delivery of their newborns. This finding is in agreement with those of a Ugandan cross-sectional study that found an inverse relationship between household socio-economic status and exclusive breastfeeding of infants when they utilized the 2006 UDHS data, however in the same study when the authors used the 2011 UDHS data, no association was found between household socio-economic status and exclusive breastfeeding among infants, contrary to the findings of this study [24]. The inverse association between household socio-economic status and exclusive breastfeeding among infants under-five months of age might be linked to the inability of mothers in the less affluent families to have access to commercial baby foods, availability of time for breastfeeding their infants since most of these mother-infant dyads live in rural areas where the predominant economic activity is agriculture and mothers tend to take their infants to their gardens (work place). The implementation of the baby friendly hospital initiative through lower level health facilities in rural areas and the WHO code of marketing of breast milk substitutes by Ugandan ministry of health might be some of the likely factors accounting for the higher likelihood of exclusive breastfeeding among infants in less affluent families, however mothers in affluent families might have all resources and access to information on breastmilk substitutes and since most of these mothers live in urban areas and tend to either work in offices or engaged in commercial activities, they tend to live their infants at home taken care of by maids, hence less likely to exclusively breastfeed them in the first six months of their infants lives. Other likely explanation could be linked to the limited period of maternity leave, often three months and lack of appropriate breastfeeding facilities in most working places in urban areas of Uganda, although probable it might warrant further investigations.

\section{Conclusion}

An inverse relationship was found between household socio-economic status and exclusive breastfeeding of infants below six months of age. However inadequate evidence was found to conclude existence of an association between maternal socio-economic status and exclusive breastfeeding of infants below six months of age. Targeting exclusive breastfeeding interventions to mother-infant dyad living in affluent families might be important in reducing socioeconomic inequality in exclusive breastfeeding among infants below six months of age in Uganda. Further studies might be required to investigate the relationship between maternal occupation and exclusive breastfeeding at regional levels.

\section{Strengths and Weakness}

This study is based on a nationally representative survey hence the findings can be generalized to all infants in
Uganda. Mothers might have only reported one form of occupation yet it's a common practice for women in Uganda to engage in two or more occupations to earn living. This might have led to misclassification of mothers with respect to the exposure (occupation) leading to under or overestimation of the effect measure. Due to small number of subjects in some categories of maternal occupation, we regrouped subjects from two or more occupational groups into one category; this might have led to residual confounding. Other potential confounders might not have been adjusted for, leading to over or underestimate of the effect of socioeconomic status on exclusive breastfeeding.

\section{Acknowledgements}

We acknowledge the management of Victoria University Kampala for providing an enabling environment for scientific research.

\section{Conflict of Interest}

All the authors do not have any possible conflicts of interest.

\section{References}

[1] Motee, A. and R. Jeewon, Importance of exclusive breastfeeding and complementary feeding among infants. Current Research in Nutrition and Food Science Journal, 2014. 2(2): p. 56-72.

[2] Victora, C. G. et al, Breastfeeding in the 21st century: epidemiology, mechanisms, and lifelong effect. The Lancet, 2016. 387(10017): p. 475-490.

[3] Sankar, M. J, et al, Optimal breastfeeding practices and infant and child mortality: a systematic review and meta - analysis. Acta paediatrica, 2015. 104: p. 3-13.

[4] Horta, B. L, et al, Evidence on the long-term effects of breastfeeding: systematic review and meta-analyses. 2007.

[5] Unicef, Innocenti Declaration on the Protection, Promotion and Support of Breastfeeding, 1 August 1990, Florence, Italy. 1990: Unicef.

[6] Kramer, M. S. and R. Kakuma, The optimal duration of exclusive breastfeeding, in Protecting infants through human milk. 2004, Springer. p. 63-77.

[7] Khanal, P, Millenium Development Goals. Health Prospect, 2011. 10: p. 57-60.

[8] 2018, U. W, Global breastfeeding scorecard 2018. 2018.

[9] Issaka, A. I, K. E. Agho, and A. M. Renzaho, Prevalence of key breastfeeding indicators in 29 sub-Saharan African countries: a meta-analysis of demographic and health surveys (2010-2015). BMJ open, 2017. 7(10): p. e014145.

[10] UNICEF, Tracking progress for breastfeeding policies and programmes: global breastfeeding scorecard 2017. Geneva, Switzerland: World Health Organization, 2017. 
[11] ICF, U. B. o. S. U. and H. Survey, Uganda Demographic Health Survey 2016. 2016.

[12] Organization, W. H, World Health Statistics 2016: Monitoring Health for the Sustainable Development Goals (SDGs). 2016: World Health Organization.

[13] Branca, F, et al, Extension of the WHO maternal, infant and young child nutrition targets to 2030. SCN News, 2015(41): p. 55-58.

[14] Ismail, T, et al, The extended Theory of Planned Behavior in explaining exclusive breastfeeding intention and behavior among women in Kelantan, Malaysia. Nutrition research and practice, 2016. 10(1): p. 49-55.

[15] Swanson, V. and K. G. Power, Initiation and continuation of breastfeeding: theory of planned behaviour. Journal of advanced nursing, 2005. 50(3): p. 272-282.

[16] Amir, L, Social theory and infant feeding. 2011, BioMed Central.

[17] Hale, J. L, B. J. Householder, and K. L. Greene, The theory of reasoned action. The persuasion handbook: Developments in theory and practice, 2002. 14: p. 259-286.

[18] Marmot, M, et al, The Marmot review: Fair society, healthy lives. The Strategic Review of Health Inequalities in England Post-2010, 2010.

[19] Ajetunmobi, T. and B. Whyte, GCPH Breastfeeding Project: Investigation of breastfeeding rates in deprived areas. Literature review. 2012, Glasgow: GCPH.

[20] Mututho, L. N, W. K. Kiboi, and P. K. Mucheru, Factors associated with exclusive breastfeeding in Kenya: a systematic review. International Journal of Community Medicine and Public Health, 2017. 4(12): p. 4358-4362.

[21] Setegn, T, et al, Factors associated with exclusive breastfeeding practices among mothers in Goba district, south east Ethiopia: a cross-sectional study. International breastfeeding journal, 2012. 7(1): p. 17.

[22] Onah, S, et al, Infant feeding practices and maternal sociodemographic factors that influence practice of exclusive breastfeeding among mothers in Nnewi South-East Nigeria: a cross-sectional and analytical study. International breastfeeding journal, 2014. 9(1): p. 6.

[23] Howe, L. D, et al, Measuring socio-economic position for epidemiological studies in low-and middle-income countries: a methods of measurement in epidemiology paper. International journal of epidemiology, 2012. 41(3): p. 871886 .
[24] Ickes, S. B, T. E. Hurst, and V. L. Flax, Maternal literacy, facility birth, and education are positively associated with better infant and young child feeding practices and nutritional status among Ugandan children. The Journal of nutrition, 2015. 145(11): p. 2578-2586.

[25] Bbaale, E, Determinants of early initiation, exclusiveness, and duration of breastfeeding in Uganda. Journal of health, population, and nutrition, 2014. 32(2): p. 249.

[26] Ssenyonga, R, R. Muwonge, and I. Nankya, Towards a better understanding of exclusive breastfeeding in the era of HIV/AIDS: a study of prevalence and factors associated with exclusive breastfeeding from birth, in Rakai, Uganda. Journal of tropical pediatrics, 2004. 50(6): p. 348-353.

[27] Azeze, G. A, et al, Exclusive Breastfeeding Practice and Associated Factors among Mothers in Boditi Town, Wolaita Zone, Southern Ethiopia, 2018: A Community-Based CrossSectional Study. International Journal of Pediatrics, 2019. 2019.

[28] Tan, K. L, Factors associated with exclusive breastfeeding among infants under six months of age in peninsular Malaysia. International breastfeeding journal, 2011. 6(1): p. 2.

[29] Zhang, Z, et al, What factors influence exclusive breastfeeding based on the theory of planned behaviour. Midwifery, 2018. 62: p. 177-182.

[30] Statistics, U. B. o, Population Projections 2015-2020. 2015.

[31] Organization, W. H, Indicators for assessing infant and young child feeding practices: part 2: measurement. 2010.

[32] Commission, U. A, The Uganda HIV and AIDS Country Progress Report. Kampala: Uganda AIDS Commission, 2016.

[33] Uganda, M, Policy Guidelines on Infant and Young Child Feeding. January 2009. 2009.

[34] Elias, P, Occupational classification (ISCO-88). 1997.

[35] Organization, W. H, Indicators for assessing infant and young child feeding practices: conclusions of a consensus meeting held 6-8 November 2007 in Washington DC, USA. 2008: World Health Organization (WHO).

[36] Demographic, K, Health Survey 2014: key indicators. Kenya National Bureau of Statistics (KNBS) and ICF Macro, 2014.

[37] MoHCDGEC, M, NBS, Office of the Chief Government Statistician, \& ICF. Demographic and health survey and malaria indicator survey (TDHS-MIS), 2015. 16.

[38] Organization, I. L, Women and men in the informal economy. A statistical picture. 2002: International Labour Organization. 